\title{
A comparison between the low back pain scales for patients with lumbar disc herniation: validity, reliability, and responsiveness
}

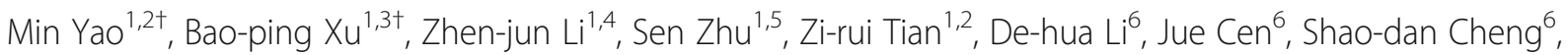
Yong-jun Wang ${ }^{1,2}$, Yan-ming Guo ${ }^{6 *}$ and Xue-jun Cui ${ }^{1,2^{*}}$ D

\begin{abstract}
Background: Although the Japanese Orthopedic Association Back Pain Evaluation Questionnaire (JOABPEQ), Numerical Pain Rating Scale (NPRS), Oswestry Disability Index (ODI), Roland Morris Disability Questionnaire (RMDQ), and Short Form 36 Health Survey (SF-36) has shown a preferable psychometric properties in patients with low back pain (LBP), but no study has yet determined these in conservative treatment of patients with lumbar disc herniation (LDH). Thus the current study aimed to compare those scales in LDH patients receiving conservative treatment to select the better option to assess the severity of disease.
\end{abstract}

Methods: LDH patients were invited to complete the JOABPEQ, NPRS, ODI, RMDQ, and SF-36 twice. The internal consistency was evaluated by the Cronbach's a. Test-retest reliability was tested by the intraclass correlation coefficient (ICC). The relationships of these scales were evaluated by the Pearson correlation coefficients ( $r$ ). The responsiveness was operationalised using the receiver operating characteristic (ROC) curve, as well as the comparison of smallest detectable change (SDC), minimum important change (MIC).

Results: A total of $353 \mathrm{LDH}$ patients were enrolled. Four subscales of the Chinese JOABPEQ were over 0.70, then the ICCS for the test-retest reliability were over 0.75 . For functional status, remarked negative correlations could be seen between JOABPEQ Q2-Q4 and ODI, as well as RMDQ ( $r=-0.634$ to -0.752$)$. For general health status, remarkable positive correlations could also be seen between Q5 Mental health and SF-36 PCS $(r=0.724)$ as well as SF-36 MCS ( $r=0.736)$. Besides, the area under of the curves (AUC) of the JOABPEQ ranged from 0.743 to 0.827 , indicating acceptale responsiveness, as well as the NPRS, ODI, and RMDQ.

Conclusion: NPRS, and ODI or RMDQ is recommended in studies related to LDH patients, while if the quality of life also is needed to observe, the NPRS, and JOABPEQ would be more appropriate rather than SF-36.

Keywords: Responsiveness, Minimal detectable change, Minimal clinically important difference, Low Back pain, Lumbar disc herniation

\footnotetext{
*Correspondence: guoym1218@163.com; 13917715524@139.com

†Bao-ping Xu contributed equally to this work and should be considered cofirst author.

${ }^{6}$ Shanghai Guanghua Hospital, 540 Xinhua Road, Shanghai 200052, China

'Spine Disease Institute, Longhua Hospital, Shanghai University of Traditional Chinese Medicine, 725 South Wanping Road, Shanghai 200032, China

Full list of author information is available at the end of the article
}

(C) The Author(s). 2020 Open Access This article is licensed under a Creative Commons Attribution 4.0 International License, which permits use, sharing, adaptation, distribution and reproduction in any medium or format, as long as you give appropriate credit to the original author(s) and the source, provide a link to the Creative Commons licence, and indicate if changes were made. The images or other third party material in this article are included in the article's Creative Commons licence, unless indicated otherwise in a credit line to the material. If material is not included in the article's Creative Commons licence and your intended use is not permitted by statutory regulation or exceeds the permitted use, you will need to obtain permission directly from the copyright holder. To view a copy of this licence, visit http://creativecommons.org/licenses/by/4.0/ The Creative Commons Public Domain Dedication waiver (http://creativecommons.org/publicdomain/zero/1.0/) applies to the data made available in this article, unless otherwise stated in a credit line to the data. 


\section{Introduction}

Lumbar disc herniation (LDH) is one of the common causes of low back pain (LBP) $[1,2]$. Symptomatic herniations present as lumbar radiculopathy including radicular pain, sensory abnormalities from both a mechanical compression and chemical irritation of the nerve root [2]. LDH occurs in approximately $10 \%$ of the population and has a serious impact on the work and life quality of patients and is the most common causes working-age individuals to undergo lumbar spine surgery, and also generates a large economic burden [3, 4].

Nevertheless, no objective biological markers are available to evaluate LDH severity, it is well known that the patient's opinion of the results by patient-reported outcomes tools are still a very important measurement of treatment quality, several patient-reported outcomes tools were used to assess LBP such as the Numerical Pain Rating Scale (NPRS), and the Visual Analogue Scale (VAS) for pain intensity, the Roland Morris Disability Questionnaire (RMDQ), and the Oswestry Disability Index (ODI) for functional status, and the Short Form 36 Health Survey (SF-36) for general health status [5]. While the Japanese Orthopedic Association Back Pain Evaluation Questionnaire (JOABPEQ), it included five subscales including Q1 Low back pain for pain intensity, Q2 Lumbar function, Q3 Walking ability, Q4 Social life function for functional status, and Q5 Mental health for general health status, which is more comprehensive to assess pain intensity, functional status, and quality of life [6]. It was concluded that there were small correlations between JOABPEQ and NPRS, medium correlations between Q2 Lumbar function, Q3 Walking ability, Q4 Social life function and ODI, RMDQ, Short Form 8 Health Survey physical component summary (SF-8 PCS); and between Q5 Mental health and SF8, SF-36, and EuroQol-5D (EQ-5D) in LBP patients or patients after lumbar surgery [7-10].

Although all of these scales has shown a preferable psychometric properties in patients with LBP, but no study has yet determined these psychometric properties in conservative treatment of patients with $\mathrm{LDH}[7,10$, 11]. The RMDQ is comprised of 24 items, the ODI is made up of 10 items, and the SF-36 consists of 36 items, JOABPEQ with 25 items, which will undoubtedly add to the burdens on clinicians during research work. Based on the above, this current study was carried out to compare the validity, reliability, and responsiveness of the JOABPEQ, NPRS, RMDQ, ODI, and SF-36 in LDH patients receiving conservative treatment to select the better option to assess the severity of disease.

\section{Materials and methods}

Patients and setting

LDH patients were consecutively recruited from the Longhua Hospital affiliated to Shanghai University of
Traditional Chinese Medicine, and Shanghai Guanghua Hospital of Integrated Traditional Chinese and Western Medicine. To be eligible to participate in the study, participants were required to be: (1) aged 18-70 years, (2) Native Chinese speaking, (3) radiculopathy related to corresponding lumbar herniated disc with or without LBP for 1 week, radiculopathy including radicular pain, sensory abnormalities with numbness of the lower limb as the main symptom, and weakness in the distribution of one or more lumbosacral nerve roots, focal paresis, restricted trunk flexion, and increases in leg pain with straining, coughing, and sneezing are also indicative, (4) magnetic resonance imaging with single or multiple lumbar disc herniation within half a year, and (5) signed the written informed consent. Exclusion criteria included: (1) LBP with other back pathologies, such as spondylolisthesis, ankylosing spondylitis, spinal fracture, rheumatoid arthritis, secondary to tumor or other disease, (2) pregnant women, (3) patients with mental disorders, cancer and other malignant disease.

\section{Ethical considerations}

The full study protocol was approved by the Longhua Hospital Research Ethics Committee (No. 2016LCSY030). All patients participating in the study provided informed consent.

\section{Questionnaires}

A questionnaire booklet was constructed for the current study, including the Chinese JOABPEQ [7], NPRS, Chinese 24-item RMDQ [12], Chinese ODI [13], and Chinese SF-36 [14].

\section{$J O A B P E Q$}

The JOABPEQ is developed from the original Japanese Orthopedic Association (JOA) scale for assessing LBP, which is disease specific and allows for judging patient outcome and self-administration. It is made up of 25 LBP-related items classified into five multi-item subscales, namely, Q1 Low back pain, Q2 Lumbar function, Q3 Walking ability, Q4 Social life function, and Q5 Mental health. The score of each factor ranges from 0 to 100 points, and a lower score is associated with worse dysfunction [15]. The five subscale scores should be used independently; adding all or some of the five subscale scores does not make sense, and summing the subscale scores to provide a total score is not necessary. The simplified Chinese version of the JOABPEQ is a reliable and valid instrument to measure functional status in patients with LBP from previous study [7].

\section{NPRS}

The NPRS is frequently employed to measure pain intensity, in which patients are asked to select a number (from 0 to 10) to represent their pain severity [16]. 


\section{$R M D Q$}

The RMDQ is a health status measure, which is designed to be completed by patients to assess their physical disability of LBP. It consists of 24 items addressing daily life and physical activity, such as personal care, sleeping, work and walking [17]. One point is assigned to each of these items, resulting in the total scores of 0 (no disability) to 24 (maximum disability) points [12].

\section{$O D I$}

The ODI is commonly used in clinical trials to measure the functional status of patients with spinal disorders [17]. It is comprised of 10 dimensions, with 6 levels being set in each dimension. Specifically, a score of 0 represents the lowest disability level, while 5 indicates the highest disability level. Moreover, the total score is converted into percentage, with a consequent maximum of $100 \%$. Notably, version 2.1 adopted in the current study has been translated and cross-culturally adapted for Chinese patients [18].

\section{SF-36}

The SF-36 is composed of 8 multi-item scales, which can assess the physical function, role limitations due to physical health problems, bodily pain, general health, vitality, social functioning, role limitations due to emotional problems and emotional well-being of patients [14]. Specifically, these eight scales have been aggregated into two summary measures, which are the Physical Component Summary (PCS) score and Mental Component Summary (MCS) score [19].

\section{Follow-up}

The patients were asked to return to the hospitals to complete the questionnaire booklet again 7-14 days after the first interview. Subsequently, all LBP scales were assessed again. The global patient evaluation (GPE) was evaluated using a 7-point Likert scale that was also completed in the second interview [20]. Besides, the response options were designed as completely recovered, much improved, slightly improved, unchanged, slightly worsened, much worsened, and worse than ever. Such scale aimed to obtain the patient ratings of improvement/deterioration as well as the importance of changes.

\section{Data analysis}

Participants who had completed the questionnaires at baseline and 7 days later were included in the subsequent analyses. Continuous variables were summarized as the mean \pm standard deviation unless otherwise noted. Data were tabulated using Microsoft EXCEL. Statistical analyses were carried out using SPSS (Version 21.0, SPSS, Gorinchem, The Netherlands). Meanwhile, the
Bland-Altman method was implemented using the MedCalc statistical (Version 19.1.7, Amazon, UK).

\section{Internal consistency}

The internal consistency of each domain was evaluated by the Cronbach's $\alpha$. In general, a Cronbach's $\alpha$ of $>0.7$ was acceptable [21]. All the completed baseline data were included in the analysis.

\section{Test-retest reliability}

The questionnaires accomplished 7 days later was tested by the intraclass correlation coefficient (ICC) (two-way random effects model, absolute agreement). Generally, an ICC of $>0.7$ is recommended as a minimum standard for reliability [22]. Only patients that were rated "no change" in their global evaluation were included, since we did not propose to prevent the treatment for patients.

\section{Construct validity}

The relationships of the JOABPEQ, NPRS, RMDQ, ODI, and SF-36 were evaluated by means of Pearson correlation coefficients $(r)$. According to Cohen's criteria, $r=$ 0.2 can be considered a small correlation, $r=0.5$ is a medium correlation, and $r=0.8$ is a large correlation [23].

\section{Measurement error}

Standard error of measurement (SEM), smallest detectable change (SDC) and Limits of Agreement (LOA) according to Bland-Altman method were used to calculate measurement error. The SEM can indicate the precision of outcome measure, which can be estimated by taking the square root of the within-subject variance of patients categorized as "unchanged" on the GPE. The SDC is calculated in accordance with $1.96 * \sqrt{ } 2 * \mathrm{SEM}$, which can be $95 \%$ confident that the observed change is a real change that is not caused by measurement error. The observed change represents the result of 2 measurements at baseline and follow-up, which therefore occurs twice, hence $\sqrt{ } 2$ [24]. The LoA was performed using Bland-Altman method, where the difference between baseline and final scores (in Y-axis) were plotted against the mean of each score at baseline and final measurement (in X-axis) [25].

\section{Minimum important change (MIC)}

The MIC is defined as the minimal threshold of perceptible symptom improvement that is considered as meaningful by the patients [26]. Subsequently, patients were divided into two groups based on the GPE of 7-point Likert scale, namely, the slightly improved or unchanged groups. Thus, the mean change score between two groups for the smallest meaningful change was taken as the MIC [27]. 


\section{Responsiveness}

The responsiveness has been defined as the ability of a questionnaire to detect the clinically important changes over time, even though these changes are small [28]. The responsiveness of the JOABPEQ was assessed by receiver operating characteristic (ROC) curve. In terms of the ROC curve, patients were dichotomized into four groups based on the GPE of 7point Likert scale as completely recovered, much improved, slightly improved, or unchanged. The sensitivity values and false-positive rates (1-specificity) were plotted on the $\mathrm{Y}$ - and the $\mathrm{X}$-axis of the curve, respectively. The area under the curve (AUC) represented the probability that a measure could correctly classify patients as clinically important improved or unchanged. An AUC of 0.7-0.8 was considered as acceptable and that of $0.8-0.9$ as excellent [29].

\section{Results}

Patient characteristics

A total of $353 \mathrm{LDH}$ patients were enrolled during a 12month period. The mean age of patients was $50.53 \pm$ 13.48 years and over $55 \%$ were female. The duration of the disease was $261.28 \pm 327.53$ weeks, 319 (90.37\%) out of those 353 patients reported low back pain, 322 (91.22\%) patients reported leg pain, 203 (57.51\%) patients reported numbness of lower limb, 64 (18.13\%) patients reported weakness of lower limb. Over half of the patients with L4/L5 level herniation (236/66.86\%), and L5/S1 level herniation (195/55.24\%). The baseline patient characteristics were shown in Table 1.

Finally, a total of 329 patients had completed the questionnaires twice at an interval of $8.87 \pm 2.70$ days, resulting in the response rate of 93.2\%. Among them, 19 patients were rated as "completely recovered", 90 as "much improved", 139 as "slightly improved", 65 as

Table 1 Characteristics of patients with LDH

\begin{tabular}{|c|c|c|c|}
\hline Characteristic & Baseline Group $(\boldsymbol{n}=353)$ & Small improve Group $(\boldsymbol{n}=139)$ & No change Group $(\boldsymbol{n}=65)$ \\
\hline Age, years & $50.53 \pm 13.48$ & $51.64 \pm 13.29$ & $50.13 \pm 14.78$ \\
\hline Gender, male/female & $156 / 197$ & $62 / 77$ & $33 / 32$ \\
\hline Disease duration, weeks & $261.28 \pm 327.53$ & $265.19 \pm 327.92$ & $370.07 \pm 408.96$ \\
\hline Occupation, active/retired & $94 / 259$ & $41 / 98$ & $15 / 49$ \\
\hline Low back pain, $n(\%)$ & $319(90.37 \%)$ & 125 (89.93\%) & $60(92.31 \%)$ \\
\hline Leg pain, $n(\%)$ & $322(91.22 \%)$ & $126(90.65 \%)$ & $57(87.69 \%)$ \\
\hline Numbness of lower limb, $n$ (\%) & $203(57.51 \%)$ & $75(53.96 \%)$ & $39(60.00 \%)$ \\
\hline Weakness of lower limb, $n$ (\%) & $64(18.13 \%)$ & $21(15.11 \%)$ & $19(29.23 \%)$ \\
\hline \multicolumn{4}{|l|}{ Lesion location, $n$ (\%) } \\
\hline $\mathrm{L} 1 / \mathrm{L} 2$ & $8(2.27 \%)$ & $3(2.16 \%)$ & $0(00.00 \%)$ \\
\hline $\mathrm{L} 2 / \mathrm{L} 3$ & $18(5.10 \%)$ & $7(5.04 \%)$ & $2(3.08 \%)$ \\
\hline $\mathrm{L} 3 / \mathrm{L} 4$ & $57(16.15 \%)$ & $25(17.99 \%)$ & $16(24.62 \%)$ \\
\hline L4/L5 & $236(66.86 \%)$ & $97(69.78 \%)$ & $49(75.38 \%)$ \\
\hline$L 5 / S 1$ & 195 (55.24\%) & $83(59.71 \%)$ & $44(67.69 \%)$ \\
\hline \multicolumn{4}{|l|}{ JOABPEQ } \\
\hline Q1 Low back pain & $31.10 \pm 26.67$ & $30.24 \pm 26.16$ & $38.55 \pm 30.21$ \\
\hline Q2 Lumbar function & $44.59 \pm 27.52$ & $43.30 \pm 27.07$ & $53.65 \pm 28.52$ \\
\hline Q3 Walking ability & $56.62 \pm 30.46$ & $54.92 \pm 30.49$ & $65.40 \pm 29.98$ \\
\hline Q4 Social life function & $35.88 \pm 21.40$ & $36.15 \pm 21.03$ & $41.01 \pm 21.89$ \\
\hline Q5 Mental health & $42.56 \pm 18.88$ & $41.52 \pm 18.89$ & $43.22 \pm 17.30$ \\
\hline NPRS & $5.68 \pm 2.19$ & $6.07 \pm 1.75$ & $4.37 \pm 2.06$ \\
\hline ODI & $49.57 \pm 22.47$ & $51.44 \pm 21.84$ & $45.39 \pm 24.01$ \\
\hline RMDQ & $13.31 \pm 6.45$ & $13.58 \pm 6.45$ & $10.89 \pm 7.07$ \\
\hline SF-36 PCS & $40.56 \pm 20.47$ & $40.08 \pm 20.10$ & $45.94 \pm 21.52$ \\
\hline SF-36 MCS & $50.84 \pm 21.69$ & $50.07 \pm 21.59$ & $51.90 \pm 19.87$ \\
\hline
\end{tabular}

LDH Lumbar disc herniation, JOABPEQ Japanese Orthopaedic Association Back Pain Evaluation Questionnaire, NPRS Numerical Pain Rating Scale, ODI Oswestry Disability Index, RMDQ Roland-Morris Disability Questionnaire, SF-36 Short Form Health Survey, PCS Physical Component Summary score, MCS Mental Component Summary score 


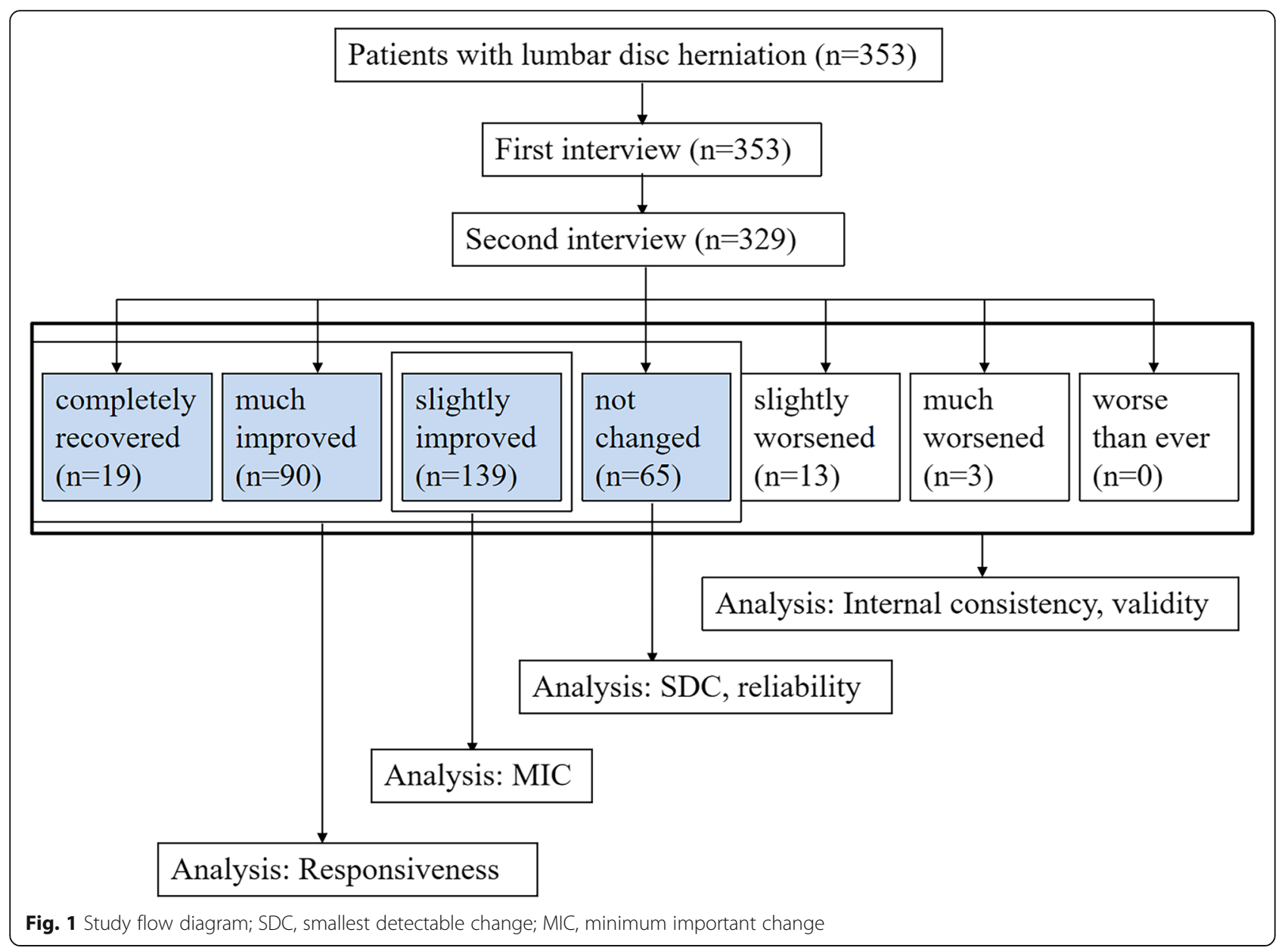

"unchanged", 13 as "slightly worsened", and 3 as "much worsened". Meanwhile, no patient was rated as "worse than ever". The demographic characteristics of patients were presented in Table 1, and the study flow diagram in Fig. 1.

\section{Data analysis \\ Internal consistency}

$353 \mathrm{LDH}$ patients were enrolled in the internal consistency, four subscales of the Chinese JOABPEQ over 0.70 (Q1 Low back pain Cronbach's $\alpha=0.494$, Q2 Lumbar function Cronbach's $\alpha=0.768$, Q3 Walking ability Cronbach's $\alpha=0.741$, Q4 Social life function Cronbach's $\alpha=0.701$, Q5 Mental health Cronbach's $\alpha=$ 0.879 ), indicating acceptable internal consistency, as well as other scales (ODI Cronbach's $\alpha=0.828$, RMDQ Cronbach's $\alpha=0.807$, SF-36 PCS Cronbach's $\alpha=0.774$, SF-36 MCS Cronbach's $\alpha=0.802$ ) (Table 2).

\section{Test-retest reliability}

65 patients showed no change were included in the testretest analysis. The ICCs for the test-retest reliability were over 0.75 (Q1 Low back pain $\mathrm{ICC}=0.751$, Q2
Table 2 The Crobach's a, and ICC for the low back pain scales

\begin{tabular}{llll}
\hline & Number of item & Crobach's a & ICC \\
\hline JOABPEQ & & & \\
Q1 Low back pain & 4 & 0.494 & 0.751 \\
Q2 Lumbar function & 6 & 0.768 & 0.809 \\
Q3 Walking ability & 5 & 0.741 & 0.812 \\
Q4 Social life function & 4 & 0.701 & 0.832 \\
Q5 Mental health & 7 & 0.879 & 0.866 \\
NPRS & 1 & $/$ & 0.991 \\
ODI & 10 & 0.828 & 0.871 \\
RMDQ & 24 & 0.807 & 0.855 \\
SF-36 PCS & 21 & 0.774 & 0.896 \\
SF-36 MCS & 14 & 0.802 & 0.843 \\
\hline
\end{tabular}

ICC intraclass correlation coefficient, JOABPEQ Japanese Orthopaedic Association Back Pain Evaluation Questionnaire, NPRS Numerical Pain Rating Scale, $O D I$ Oswestry Disability Index, RMDQ Roland-Morris Disability Questionnaire, SF-36 Short Form Health Survey, PCS Physical Component Summary score, MCS Mental Component Summary score 
Lumbar function ICC $=0.809$, Q3 Walking ability ICC $=$ 0.812 , Q4 Social life function $\mathrm{ICC}=0.832$, Q5 Mental health $\mathrm{ICC}=0.866$ ), indicating good test-retest reliability. The other scale had similar good test-retest reliability $($ NPRS ICC $=0.991$, ODI ICC $=0.871$, RMDQ ICC $=$ 0.855 , SF-36 PCS ICC $=0.896$, SF-36 MCS ICC $=0.843$ ) (Table 2).

\section{Construct validity}

There were small correlations between the NPRS, and JOABPEQ $(r=-0.388$ to -0.457$)$, similar with NPRS and ODI $(r=-0.449)$, RMDQ $(r=-0.485)$, and SF-36 MCS $(r=-0.413)$. Medium correlation correlations could be seen between JOABPEQ and ODI, as well as RMDQ (ODI $r=-0.537$ to -0.725 ; RMDQ $r=-0.597$ to -0.752 ), especially Q3 Walking ability and Q4 Social life function. Similar, medium correlations could also be seen between JOABPEQ and SF-36 PCS ( $r=0.604$ to 0.730$)$ as well as SF-36 MCS ( $r=0.517$ to 0.736$)$, especially Q5 Mental health. The Pearson correlation coefficient $(r)$ of each scale were in Table 3.

\section{Measurement error}

Sixty-five patients rated as "unchanged" were enrolled into measurement error analysis. The results suggested that the SDCs of Q1-Q5 Mental Health were ranged from 4.29 to 8.14 from SEM, then the SDCs of NPRS, ODI, RMDQ, SF-36 PCS and SF-36 MCS were 0.10,
$3.43,0.77,3.41$, and 3.93, respectively. The SDCs of the LBP scales were presented in Table 3.

All those 65 patients were enrolled into the BlandAltman plot, the LoAs of each LBP scales were in the Fig. 2. The LoAs of JOABPEQ ranged from 5.70 to 11.05 (Q1 Low back pain 11.05, Q2 Lumbar function 11.15, Q3 Walking ability 10.25, Q4 Social life function 6.65; Q5 Mental health 5.7. the SDCs of NPRS, ODI, RMDQ, SF-36 PCS and SF-36 MCS were 0.56, 5.0, 2.95, 6.9, and 7.1 , respectively.

\section{MICs}

Sixty-five patients rated as "unchanged" and 139 as "slightly improved" were enrolled in MCID analysis. The results suggested that the MICs of JOABPEQ Q1-5 were 11.37 [95\%CI 8.76, 13.97], 11.14 [8.69, 13.60], 11.18 [8.64, $13.72], 6.88$ [5.08, 8.68], and 6.17 [4.75, 7.59], respectively.

Meanwhile, the MICs of RMDQ, ODI, NPRS, SF-36 PCS and SF-36 MCS were also calculated. The results revealed that the MICs of NPRS, ODI, RMDQ, SF-36 PCS and SF-36 MCS were 1.71 [1.52, 1.91], 5.88 [4.44, 7.31], 1.74 [1.11, 2.37], 4.57 [3.33, 5.82], and 3.59 [2.58, 4.62], respectively. The MIC and MIC\% of the LBP scales were shown in Table 4.

\section{Responsiveness}

The AUCs for the responsiveness of JOABPEQ scales were presented in Table 5, and Fig. 3. As could be

Table 3 Pearson correlation coefficient $(r)$ between each low back pain scales

\begin{tabular}{|c|c|c|c|c|c|c|c|c|c|c|}
\hline Scales & $\begin{array}{l}\text { Q1 Low back } \\
\text { pain }\end{array}$ & $\begin{array}{l}\text { Q2 Lumbar } \\
\text { function }\end{array}$ & $\begin{array}{l}\text { Q3 Walking } \\
\text { ability }\end{array}$ & $\begin{array}{l}\text { Q4 Social life } \\
\text { function }\end{array}$ & $\begin{array}{l}\text { Q5 Mental } \\
\text { health }\end{array}$ & NPRS & ODI & RMDQ & $\begin{array}{l}\text { SF-36 } \\
\text { PCS }\end{array}$ & $\begin{array}{l}\text { SF-36 } \\
\text { MCS }\end{array}$ \\
\hline \multicolumn{11}{|l|}{ JOABPEQ } \\
\hline $\begin{array}{l}\text { Q1 Low back } \\
\text { pain }\end{array}$ & 1 & $0.556^{\mathrm{a}}$ & $0.523^{a}$ & $0.580^{\mathrm{a}}$ & $0.496^{a}$ & $-0.415^{a}$ & $-0.537^{a}$ & $\overline{0.627^{\mathrm{a}}}$ & $0.659^{a}$ & $0.517^{a}$ \\
\hline $\begin{array}{l}\text { Q2 Lumbar } \\
\text { function }\end{array}$ & $0.556^{\mathrm{a}}$ & 1 & $0.569^{a}$ & $0.679^{\mathrm{a}}$ & $0.556^{\mathrm{a}}$ & $-\overline{0.429^{a}}$ & $\overline{0.634^{\mathrm{a}}}$ & $\overline{0.751^{\mathrm{a}}}$ & $0.701^{a}$ & $0.553^{\mathrm{a}}$ \\
\hline $\begin{array}{l}\text { Q3 Walking } \\
\text { ability }\end{array}$ & $0.523^{\mathrm{a}}$ & $0.569^{\mathrm{a}}$ & 1 & $0.736^{\mathrm{a}}$ & $0.535^{\mathrm{a}}$ & $-0.426^{a}$ & $\overline{0.701^{\mathrm{a}}}$ & $\overline{0.725^{\mathrm{a}}}$ & $0.604^{\mathrm{a}}$ & $0.536^{\mathrm{a}}$ \\
\hline $\begin{array}{l}\text { Q4 Social life } \\
\text { function }\end{array}$ & $0.580^{\mathrm{a}}$ & $0.679^{\mathrm{a}}$ & $0.736^{\mathrm{a}}$ & 1 & $0.630^{\mathrm{a}}$ & $-0.457^{a}$ & $\overline{0.715^{\mathrm{a}}}$ & $\overline{0.724^{\mathrm{a}}}$ & $0.730^{a}$ & $0.636^{a}$ \\
\hline $\begin{array}{l}\text { Q5 Mental } \\
\text { health }\end{array}$ & $0.496^{a}$ & $0.556^{\mathrm{a}}$ & $0.535^{\mathrm{a}}$ & $0.630^{\mathrm{a}}$ & 1 & $-0.388^{a}$ & $\overline{0.587^{\mathrm{a}}}$ & $\overline{0.597^{\mathrm{a}}}$ & $0.724^{a}$ & $0.736^{a}$ \\
\hline NPRS & $-0.415^{\mathrm{a}}$ & $-0.429^{a}$ & $-0.426^{a}$ & $-0.457^{a}$ & $-0.388^{a}$ & 1 & $0.449^{a}$ & $0.485^{\mathrm{a}}$ & $-0.519^{a}$ & $-0.413^{a}$ \\
\hline ODI & $-0.537^{\mathrm{a}}$ & $-0.634^{a}$ & $-0.725^{\mathrm{a}}$ & $-0.715^{\mathrm{a}}$ & $-0.587^{a}$ & $0.449^{a}$ & 1 & $0.736^{\mathrm{a}}$ & $-0.682^{a}$ & $-0.601^{a}$ \\
\hline RMDQ & $-0.627^{a}$ & $-0.752^{\mathrm{a}}$ & $-0.701^{a}$ & $-0.724^{\mathrm{a}}$ & $-0.597^{\mathrm{a}}$ & $0.485^{\mathrm{a}}$ & $0.736^{a}$ & 1 & $-0.764^{a}$ & $-0.614^{a}$ \\
\hline SF-36 PCS & $0.659^{a}$ & $0.701^{a}$ & $0.604^{\mathrm{a}}$ & $0.730^{a}$ & $0.724^{a}$ & $-0.519^{a}$ & $-0.682^{a}$ & $\overline{0.764^{\mathrm{a}}}$ & 1 & $0.779^{a}$ \\
\hline SF-36 MCS & $0.517^{\mathrm{a}}$ & $0.553^{\mathrm{a}}$ & $0.536^{\mathrm{a}}$ & $0.636^{\mathrm{a}}$ & $0.736^{a}$ & $-0.413^{a}$ & $-0.601^{a}$ & $\overline{0.614^{\mathrm{a}}}$ & $0.770^{\mathrm{a}}$ & 1 \\
\hline
\end{tabular}

JOABPEQ Japanese Orthopaedic Association Back Pain Evaluation Questionnaire, NPRS Numerical Pain Rating Scale, ODI Oswestry Disability Index, RMDQ RolandMorris Disability Questionnaire, SF-36 Short Form Health Survey, PCS Physical Component Summary score, MCS Mental Component Summary score

According to Cohen's criteria, $r=0.2$ can be considered a small correlation, $r=0.5$ is a medium correlation, and $r=0.8$ is a large correlation

Notes

${ }^{\text {a }}$ Correlation is significant at the 0.01 level (2-tailed) 


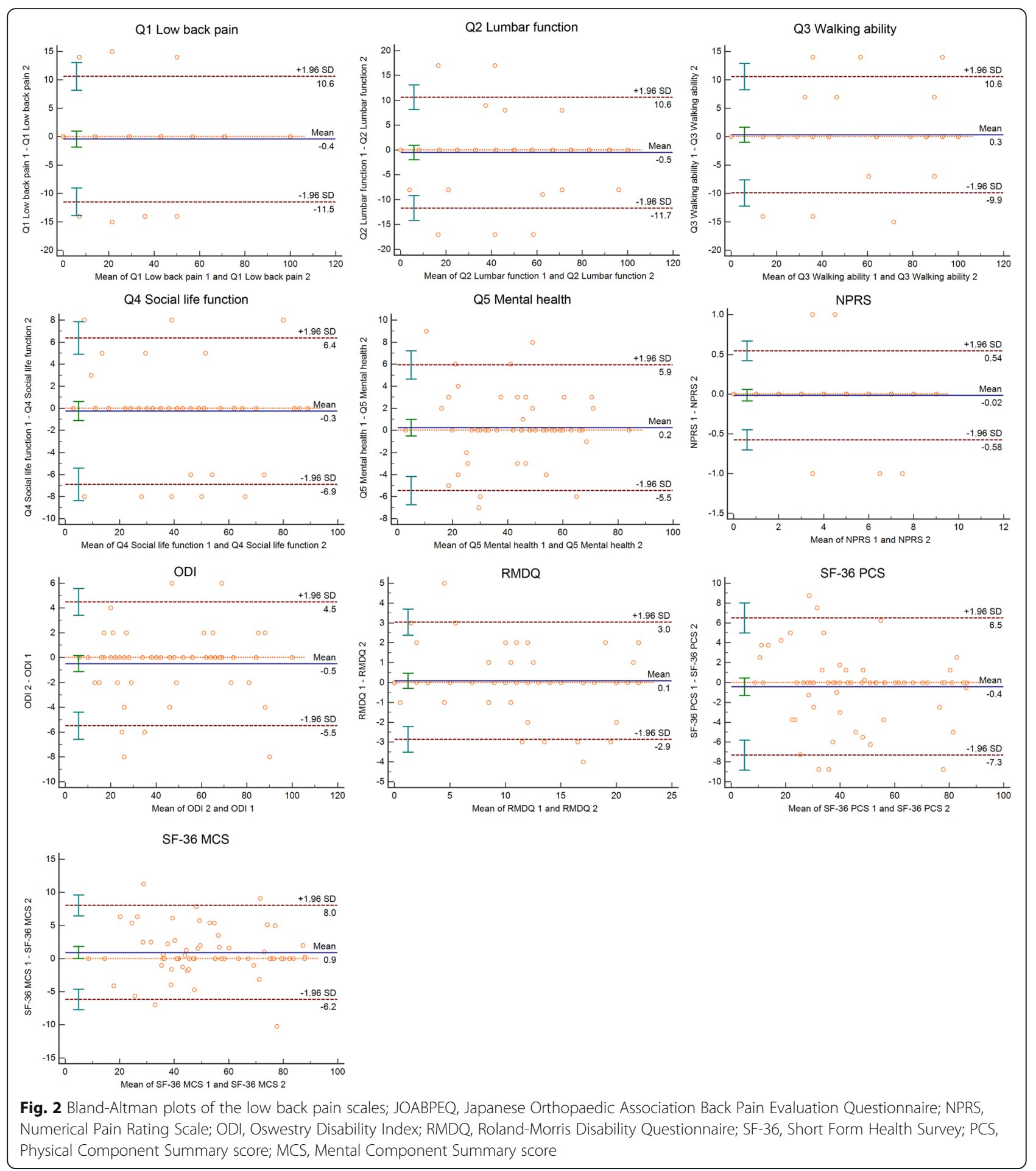

observed, the AUCs for JOABPEQ were ranged from 0.743 to 0.827 . The results of AUC indicated that the JOABPEQ Q1 had excellent responsiveness to assess pain intensity, and JOABPEQ Q2-4 had acceptable to excellent responsiveness to assess functional status. Meanwhile, the AUCs for the responsiveness of ODI and RMDQ were over 0.80 , which demonstrated excellent responsiveness to assess functional status. In addition, the AUC of NPRS was 0.880, representing excellent responsiveness to assess pain intensity; whereas those of SF-36 PCS and SF-36 MCS were 0.757 and 0.753 , respectively, suggesting acceptable ability to discriminate the patients who improved and who did not related to assess quality of life. 
Table 4 The SEM, SDC, MIC, and MIC\% for the low back pain scales

\begin{tabular}{|c|c|c|c|c|c|}
\hline Scales & Total score & SEM & SDC & MIC & MIC\% \\
\hline \multicolumn{6}{|l|}{ JOABPEQ } \\
\hline Q1 Low back pain & 100 & 2.94 & 8.14 & $11.37[8.76,13.97]$ & $37.60 \%$ \\
\hline Q2 Lumbar function & 100 & 2.38 & 6.59 & $11.14[8.69,13.60]$ & $25.73 \%$ \\
\hline Q3 Walking ability & 100 & 2.40 & 6.62 & $11.18[8.64,13.72]$ & $20.36 \%$ \\
\hline Q4 Social life function & 100 & 1.59 & 4.40 & $6.88[5.08,8.68]$ & $19.03 \%$ \\
\hline Q5 Mental health & 100 & 1.55 & 4.29 & $6.17[4.75,7.59]$ & $14.86 \%$ \\
\hline NPRS & 10 & 0.04 & 0.10 & $1.71[1.52,1.91]$ & $28.17 \%$ \\
\hline ODI & 100 & 1.25 & 3.43 & $5.88[4.44,7.31]$ & $11.43 \%$ \\
\hline RMDQ & 24 & 0.28 & 0.77 & $1.74[1.11,2.37]$ & $33.65 \%$ \\
\hline SF-36 PCS & 100 & 1.24 & 3.41 & $4.57[3.33,5.82]$ & $11.40 \%$ \\
\hline SF-36 MCS & 100 & 1.42 & 3.93 & $3.59[2.58,4.62]$ & $7.17 \%$ \\
\hline
\end{tabular}

SEM standard error of measurement, SDC smallest detectable change, MIC minimum important change, JOABPEQ Japanese Orthopaedic Association Back Pain Evaluation Questionnaire, NPRS Numerical Pain Rating Scale, ODI Oswestry Disability Index, RMDQ Roland-Morris Disability Questionnaire, SF-36 Short Form Health Survey, PCS Physical Component Summary score, MCS Mental Component Summary score

\section{Discussion}

\section{Study summary of this study}

To the best of our knowledge, the current study is the first to test the validity, reliability, and responsiveness of the JOABPEQ, NPRS, RMDQ, ODI, and SF-36 for LDH patients receiving conservative treatment. The selection for the questionnaires in this study was based on the characteristic of LBP. The main complain of LDH are LBP, disability, and impact to the life quality. The NPRS we chose is focused on pain intensity, ODI and RMDQ are disability, and the SF-36 is the most common scale to assess quality of life. The validity, reliability, and good sensitivity of NPRS has been identified in plenty of clinical trials $[16,30,31]$. To some extent, the NPRS is

Table $\mathbf{5}$ The result of the ROC analysis for the low back pain scales

\begin{tabular}{llll}
\hline Scales & AUC & Sensitivity & Specificity \\
\hline JOABPEQ & & & \\
Q1 Low back pain & $0.823[0.731,0.915]$ & 0.750 & 0.791 \\
Q2 Lumbar function & $0.743[0.619,0.868]$ & 0.600 & 0.815 \\
Q3 Walking ability & $0.808[0.719,0.898]$ & 0.850 & 0.709 \\
Q4 Social life function & $0.827[0.707,0.946]$ & 0.600 & 0.920 \\
Q5 Mental health & $0.807[0.688,0.927]$ & 0.850 & 0.682 \\
NPRS & $0.880[0.827,0.934]$ & 0.850 & 0.771 \\
ODI & $0.847[0.741,0.952]$ & 0.850 & 0.767 \\
RMDQ & $0.868[0.774,0.962]$ & 0.950 & 0.746 \\
SF-36 PCS & $0.757[0.635,0.878]$ & 0.700 & 0.812 \\
SF-36 MCS & $0.753[0.632,0.874]$ & 0.800 & 0.705 \\
\hline
\end{tabular}

$R O C$ receiver operating characteristic, $A \cup C$ area under the curve, JOABPEQ Japanese Orthopaedic Association Back Pain Evaluation Questionnaire, NPRS Numerical Pain Rating Scale, ODI Oswestry Disability Index, RMDQ RolandMorris Disability Questionnaire, SF-36 Short Form Health Survey, PCS Physical Component Summary score, MCS Mental Component Summary score superior then the other scales, such as the visual analogue scale, and verbal rating scale [31]. Then ODI, and RMDQ has been verified to be a reliable and valid LBP measurement for patients [32, 33].

It was shown that most of the scales had acceptable internal consistency, and reliability, except Q1 Low back pain in JOABPEQ. For pain intensity, small correlations between the NPRS, and other scales. For function, medium correlation could be seen between JOABPEQ and ODI, as well as RMDQ, similar, medium correlations were between JOABPEQ and SF-36 for quality of life. As the AUCs of all the scales were over 0.70, hence their responsiveness was all acceptable. In the other words, it means that for pain intensity, the NPRS could not been replaced by Q1 Low back pain, then Q2 Lumbar function, Q3 Walking ability, Q4 Social life function had the same similar performance compared with ODI, and RMDQ, then the Q5 could replace SF-36, it has higher responsiveness then SF-36, with acceptable correlation (SF-36 PCS $r=0.724$, SF-36 MCS $r=0.736$ ).

Based on the validity, reliability, and responsiveness of the LBP scales, if the studies designed to focus on pain intensity and function, the NPRS, and ODI or RMDQ are recommended, then the ODI would be more applicable to cross section survey due to a slim advantage on validity, reliability, on the contrary, RMDQ more suitable to intervention trials due to higher responsiveness in LDH patients. While if the quality of life also is needed to observe, the NPRS, and JOABPEQ would be more appropriate, as the SF36 with 36 item results to heavier workload of researchers, and patients, and harder calculation, meanwhile our results also suggest that the SF-36 displays poorer responsiveness in LDH patients compared with other scales, which is consistent with findings from other previous studies [34, 35]. More 


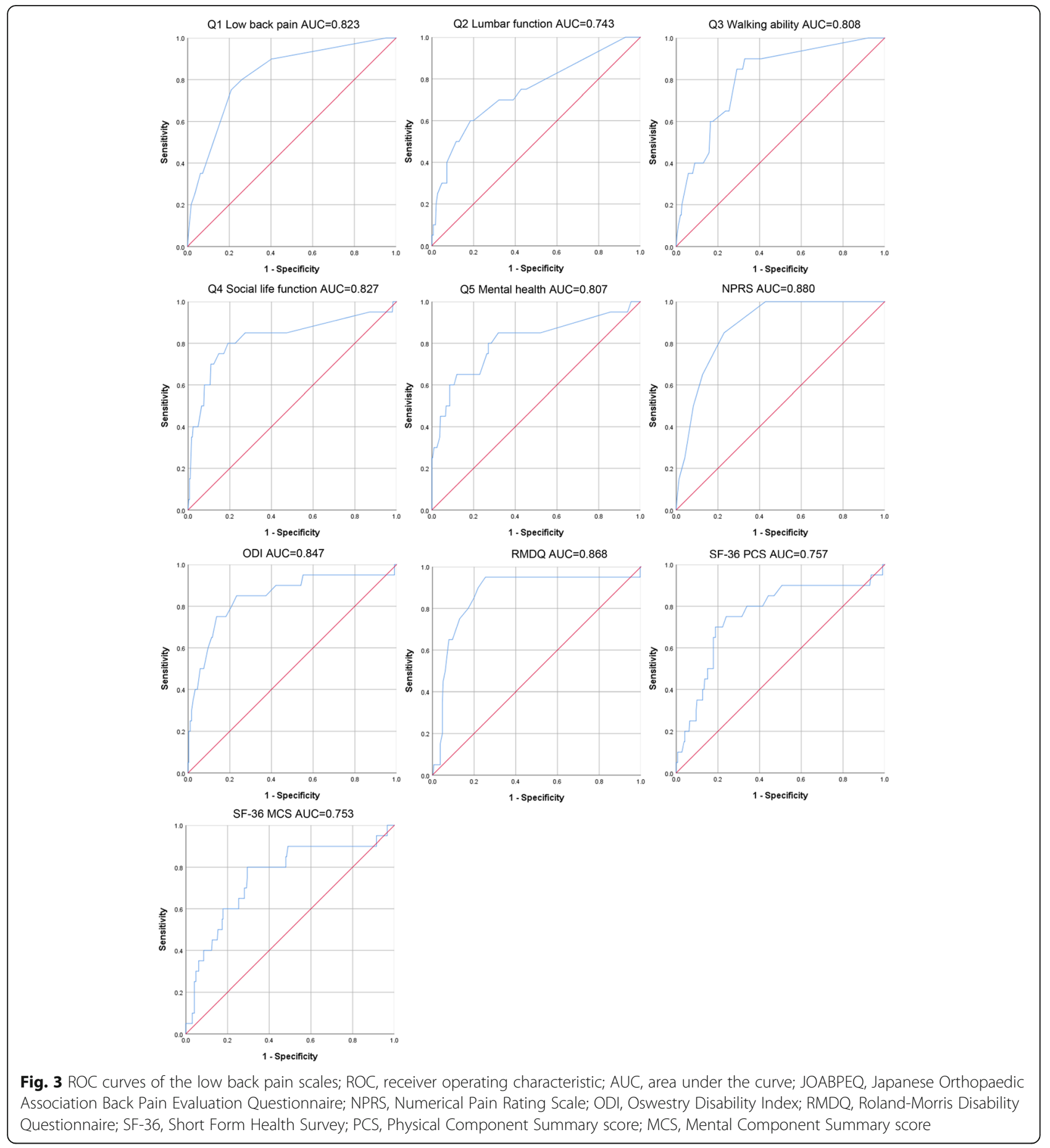

measures are also a participant burden more than clinician or researcher burden, using more scales could make the trials more expensive for the researchers, thus this recommended could give some advice on studies related to LDH to save resources and lighten the burden of researchers and participants.

\section{Validity of the LBP scales}

There were only small correlations between the NPRS, and JOABPEQ related to pain intensity, consistent with our previous research in LBP patients [7]. The NPRS is a single scale that only assesses pain intensity, then Q1 Low back pain is focused on the impact of pain to daily life, such as sleeping, and rest. Although both of them 
were designed to assess pain intensity, but with different fields. The ODI and the RMDQ are used to measure the degree of pain induced dysfunction, then Q2 Lumbar function, Q3 Walking ability, and Q4 Social life function measure the level of lumbar function, walking ability, and social life, respectively; these are important factors of pain induced dysfunction. The result was in expect that JOABPEQ has medium correlation to ODI, and RMDQ for dysfunction. The present correlations between the JOABPEQ and SF-36 are similar to those found for the Thai JOABPEQ, and our previous research in LBP patients, especially for Q5 Mental health, suggesting that this item can objectively assess the life quality of patients with LBP $[7,9]$.

\section{The MICs and responsiveness of the JOABPEQ}

Currently, the JOABPEQ has also been translated into Arabic [36], Thai [9], Turkish [8], and Persian [37]. Nonetheless, only the original version, has explored the MIC or responsiveness of the JOABPEQ. The MIC of the original JOABPEQ ranged from 27.9 points (Q3 Walking ability) to 14.8 points (Q5 Mental health) in patients with $\mathrm{LDH}$ after discectomy ([38]Azimi P 2018), then it was ranged from 28.5 points (Q1 Low back pain) to 14.5 points (Q5 Mental health) in patients with decompression surgery for lumbar spinal stenosis ([39]Ogura Y 2019). In our study, the MICs of the simplified Chinese JOABPEQ ranged from 11.37 to 6.17 points for $\mathrm{LDH}$, are smaller than the recommended by the original JOABPEQ. The trend was the same, the MICs of the Q1 to Q3 were larger than Q4 and Q5 Mental health, then the responsiveness were similar in both simplified Chinese and original version ([38] Azimi P 2018, [39] Ogura Y 2019, [40] Fujimori T). It was suspected that the patients in surgery group might had greater expectations for treatment rather than conservative treatment. In other words, the patient with higher improvement would rate slightly improved or much improved with surgery than conservative treatment. The same situation occurred in other disease, such as the American Shoulder and Elbow Surgeons (ASES) score and Simple Shoulder Test (SST) score rotator cuff disease with arthroscopic rotator cuff repair (ASES 20.9; SST 2.4) or arthroplasty (ASES 27.1; SST 4.3) or conservative treatment (ASES 12.01; SST 2.05) ([41] Tashjian RZ 2020, [42] Tashjian RZ 2016, [43] Tashjian RZ 2010). Then it was found that the patients accepted discectomy was more serious than the patients in our study, that might give the patients more space to improve. Thus the test of MIC and responsiveness is expected to be conducted in other different populations with receiving conservative treatment.

\section{Superiorities and limitations}

The use of GPE is controversial for most studies of this type, and the validity of a single-item design compared with a multi-items scale is also doubtful [44]. However, such limitations are inevitable. Moreover, the GPE is also associated with another disadvantage, which is that it may be difficult for patients to recall their initial health status and to compare it with their current status to assess any changes. Therefore, this may introduce bias [45]. In the current study, patients are asked to return to hospitals to complete the questionnaire booklet again 714 days after the first interview. This seems not to be a long time, which may be easier for patients to recall their initial health status. Secondly, treatments such as acupuncture and manual therapies are allowed for LBP patients, which have favorable short-term effects on them.

Furthermore, a small proportion of participants are retested within a short interval. Therefore, they may have been biased to give the same answer if they have remembered some of the questions asked at the first time, even though there are 96 items of these questionnaires.

\section{Conclusion}

Based on the validity, reliability, and responsiveness of the LBP scales, if the studies focus on pain intensity and function, the NPRS, and ODI for cross section survey or RMDQ intervention trials are recommended in LDH patients. While if the quality of life also is needed to observe, the NPRS, and JOABPEQ would be more appropriate rather than SF-36. This recommended could give some advice on studies related to $\mathrm{LDH}$ to save resources and lighten the burden of researchers, as well as participants.

\section{Acknowledgments}

We thank Liwen Bianji, Edanz Group China (www.liwenbianji.cn/ac), for editing the English text of a draft of this manuscript.

\section{Authors' contributions}

All authors have seen the manuscript and approved to submit to your journal. Min Yao and Bao-ping Xu conceived the study; Min Yao, Bao-ping Xu, Zhen-jun Li, Sen Zhu, Zi-rui Tian, De-hua Li, Jue Cen, Shao-dan Cheng participated in the collection of cases. Xue-jun Cui and Yong-jun Wang checked as external advisers. Min Yao and Bao-ping Xu undertook the analyses of data with Xue-jun Cui. Min Yao, Bao-ping Xu, Yan-ming Guo, Yongjun Wang and Xue-jun Cui wrote the initial draft. Xue-jun Cui and Yan-ming Guo critically revised the manuscript.

\section{Funding}

This work was supported by the National Natural Science Foundation of China (81873317, 81704096, 81603635); a grant from the Municipal Science and Technology Commission of Shanghai-TCM key project (16401970100); a grant from the Shanghai TCM Medical Center of Chronic Disease (2017ZZ01010); a grant from the National Thirteenth Five-Year Science and Technology Major Special Project for New Drug Innovation and Development (2017ZX09304001) 


\section{Ethics approval and consent to participate}

Each author certifies that Ethics committee of Longhua Hospital Research Ethics Committee (No. 2016LCSY030) approved the human protocol for this investigation, that all investigations were conducted in conformity with ethical principles of research, and that informed consent for participation in the study was obtained.

\section{Consent for publication}

All authors have agreed to publish this study.

\section{Competing interests}

The authors declare that they have no competing interests.

\section{Author details}

${ }^{1}$ Spine Disease Institute, Longhua Hospital, Shanghai University of Traditional Chinese Medicine, 725 South Wanping Road, Shanghai 200032, China. ${ }^{2}$ Key Laboratory of Theory and Therapy of Muscles and Bones, Ministry of Education, Shanghai University of Traditional Chinese Medicine, 725 South Wanping Road, Shanghai 200032, China. ' 2 u'an Hospital of Traditional Chinese Medicine, 76 Renmin Road, Anhui Lu'an 237000, China. ${ }^{4}$ Gansu Provincial Hospital of Traditional Chinese Medicine, 418 Guazhou Road, Lanzhou 730050, Gansu, China. ${ }^{5}$ Department of Orthopaedic, Shanghai Pudong Gongli Hospital, Second Military Medical University, 219 Miaopu Road, Shanghai 200013, China. '5hanghai Guanghua Hospital, 540 Xinhua Road, Shanghai 200052, China.

\section{Received: 20 June 2019 Accepted: 11 May 2020} Published online: 10 June 2020

\section{References}

1. Amin RM, Andrade NS, Neuman BJ. Lumbar disc herniation. Curr Rev Musculoskelet Med. 2017;10:507-16.

2. Omidi-Kashani F, Hasankhani EG, Moghadam MH, Esfandiari MS. Prevalence and severity of preoperative disabilities in Iranian patients with lumbar disc herniation. Arch Bone Jt Surg. 2013;1:78-81.

3. Cilingir D, Hintistan S, Yigitbas C, Nural N. Nonmedical methods to relieve low back pain caused by lumbar disc herniation: a descriptive study in northeastern Turkey. Pain Manag Nurs. 2014;15:449-57.

4. Ecklund JM, Babington PW. Lumbar disc herniation and military rank. World Neurosurg. 2014;82:e157-8.

5. Smeets R, Koke A, Lin CW, Ferreira M, Demoulin C. Measures of function in low Back pain/disorders: low Back pain rating scale (LBPRS), Oswestry disability index (ODI), progressive Isoinertial lifting evaluation (PILE), Quebec Back pain disability scale (QBPDS), and Roland-Morris disability questionnaire (RDQ). Arthritis Care Res (Hoboken). 2011;63(Suppl 11):S158-73.

6. Fukui M, Chiba K, Kawakami M, Kikuchi S, Konno S, Miyamoto M, Seichi A, Shimamura T, Shirado O, Taguchi T, et al. Japanese Orthopaedic association Back pain evaluation questionnaire. Part 3. Validity study and establishment of the measurement scale : subcommittee on low Back pain and cervical myelopathy evaluation of the clinical outcome Committee of the Japanese Orthopaedic Association, Japan. J Orthop Sci. 2008;13:173-9.

7. Yao M, Li ZJ, Zhu S, Wang JY, Pan YF, Tian ZR, Shen LY, Cheng SD, Wang YJ, Cui XJ. Simplified Chinese version of the Japanese Orthopaedic association Back pain evaluation questionnaire: cross-cultural adaptation, reliability, and validity for patients with low Back pain. Spine (Phila Pa 1976). 2018;43:E357e364.

8. Gunaydin G, Hazar Kanik Z, Karabicak GO, Sozlu U, Pala OO, Alkan ZB, Basar S, Citaker S. Cross-cultural adaptation, reliability and validity of the Turkish version of the Japanese Orthopaedic association Back pain evaluation questionnaire. J Orthop Sci. 2016;21:295-8.

9. Poosiripinyo T, Paholpak P, Jirarattanaphochai K, Kosuwon W, Sirichativapee W, Wisanuyotin T, Laupattarakasem P, Sukhonthamarn K, Jeeravipoolvarn P, Sakakibara T, Kasai Y. The Japanese orthopedic association Back pain evaluation questionnaire (JOABPEQ): a validation of the reliability of the Thai version. J Orthop Sci. 2017;22:34-7.

10. Lauridsen HH, Hartvigsen J, Manniche C, Korsholm L, Grunnet-Nilsson N. Responsiveness and minimal clinically important difference for pain and disability instruments in low back pain patients. BMC Musculoskelet Disord. 2006;7:82.

11. Childs JD, Piva SR, Fritz JM. Responsiveness of the numeric pain rating scale in patients with low back pain. Spine (Phila Pa 1976). 2005;30:1331-4.
12. Fan $\mathrm{S}, \mathrm{Hu} \mathrm{Z}$, Hong H, Zhao F. Cross-cultural adaptation and validation of simplified Chinese version of the Roland-Morris disability questionnaire. Spine (Phila Pa 1976). 2012;37:875-80.

13. Ma C, Wu S, Xiao L, Xue Y. Responsiveness of the Chinese version of the Oswestry disability index in patients with chronic low back pain. Eur Spine J. 2011;20:475-81.

14. Li L, Wang HM, Shen Y. Chinese SF-36 health survey: translation, cultural adaptation, validation, and normalisation. J Epidemiol Community Health. 2003;57:259-63.

15. Fukui M, Chiba K, Kawakami M, Kikuchi S, Konno S, Miyamoto M, Seichi A, Shimamura T, Shirado O, Taguchi T, et al. Japanese Orthopaedic association Back pain evaluation questionnaire. Part 2. Verification of its reliability : the subcommittee on low Back pain and cervical myelopathy evaluation of the clinical outcome committee of the Japanese Orthopaedic association. J Orthop Sci. 2007;12:526-32.

16. Pool JJ, Ostelo RW, Hoving JL, Bouter LM, de Vet HC. Minimal clinically important change of the neck disability index and the numerical rating scale for patients with neck pain. Spine (Phila Pa 1976). 2007;32:3047-51.

17. Roland M, Fairbank J. The Roland-Morris disability questionnaire and the Oswestry disability questionnaire. Spine (Phila Pa 1976). 2000;25:3115-24.

18. Liu H, Tao H, Luo Z. Validation of the simplified Chinese version of the Oswestry disability index. Spine (Phila Pa 1976). 2009;34:1211-6 discussion 1217.

19. Zhou KN, Zhang M, Wu Q, Ji ZH, Zhang XM, Zhuang GH. Reliability, validity and sensitivity of the Chinese (simple) short form 36 health survey version 2 (SF-36V2) in patients with chronic hepatitis B. J Viral Hepat. 2013;20:e47-55.

20. Fischer D, Stewart AL, Bloch DA, Lorig K, Laurent D, Holman H. Capturing the patient's view of change as a clinical outcome measure. Jama. 1999;282: 1157-62.

21. Terwee CB, Bot SD, de Boer MR, van der Windt DA, Knol DL, Dekker J, Bouter LM, de Vet HC. Quality criteria were proposed for measurement properties of health status questionnaires. J Clin Epidemiol. 2007;60:34-42.

22. Fitzpatrick R, Davey C, Buxton MJ, Jones DR. Evaluating patient-based outcome measures for use in clinical trials. Health Technol Assess. 1998;2(iiv):1-74.

23. Cohen J. A power primer. Psychol Bull. 1992;112:155-9.

24. Bland JM. Minimal detectable change. Phys Ther Sport. 2009;10:39 author reply $39-40$.

25. Bland JM, Altman DG. Statistical methods for assessing agreement between two methods of clinical measurement. Lancet. 1986;1:307-10.

26. Lydick E, Epstein RS. Interpretation of quality of life changes. Qual Life Res. 1993;2:221-6.

27. Hays RD, Woolley JM. The concept of clinically meaningful difference in health-related quality-of-life research. How meaningful is it? Pharmacoeconomics. 2000;18:419-23.

28. Guyatt GH, Deyo RA, Charlson M, Levine MN, Mitchell A. Responsiveness and validity in health status measurement: a clarification. J Clin Epidemiol. 1989:42:403-8.

29. Ward MM, Marx AS, Barry NN. Identification of clinically important changes in health status using receiver operating characteristic curves. J Clin Epidemiol. 2000;53:279-84

30. Williamson A, Hoggart B. Pain: a review of three commonly used pain rating scales. J Clin Nurs. 2005;14:798-804

31. Hjermstad MJ, Fayers PM, Haugen DF, Caraceni A, Hanks GW, Loge JH, Fainsinger R, Aass N, Kaasa S. European palliative care research collaborative (EPCRC): studies comparing numerical rating scales, verbal rating scales, and visual analogue scales for assessment of pain intensity in adults: a systematic literature review. J Pain Symptom Manag. 2011;41:1073-93.

32. Chiarotto A, Maxwell LJ, Terwee CB, Wells GA, Tugwell P, Ostelo RW. Roland-Morris disability questionnaire and Oswestry disability index: which has better measurement properties for measuring physical functioning in nonspecific low Back pain? Systematic Review and Meta-Analysis. Phys Ther. 2016:96:1620-37.

33. Koç M, Bayar B, Bayar K. A comparison of Back pain functional scale with Roland Morris disability questionnaire, Oswestry disability index, and short form 36-health survey. Spine. 2018;43:877-82.

34. Kopec JA, Esdaile JM. Functional disability scales for back pain. Spine (Phila Pa 1976). 1995;20:1943-9.

35. Suarez-Almazor ME, Kendall C, Johnson JA, Skeith K, Vincent D. Use of health status measures in patients with low back pain in clinical settings. Comparison of specific, generic and preference-based instruments. Rheumatology (Oxford). 2000;39:783-90. 
36. Alfayez SM, Bin Dous AN, Altowim AA, Alrabiei QA, Alsubaie BO, Awwad WM. The validity and reliability of the Arabic version of the Japanese orthopedic association Back pain evaluation questionnaire: can we implement it in Saudi Arabia? J Orthop Sci. 2017;22:618-21.

37. Azimi P, Shahzadi S, Montazeri A. The Japanese orthopedic association Back pain evaluation questionnaire (JOABPEQ) for low back disorders: a validation study from Iran. J Orthop Sci. 2012;17:521-5.

38. Azimi P, Yazdanian T, Benzel EC. Determination of minimally clinically important differences for JOABPEQ measure after discectomy in patients with lumbar disc herniation. J Spine Surg. 2018:4:102-8.

39. Ogura Y, Ogura K, Kobayashi Y, Kitagawa T, Yonezawa Y, Takahashi Y, Yoshida K, Yasuda A, Shinozaki Y, Ogawa J. Minimally clinically important differences for the Japanese Orthopaedic association Back pain evaluation questionnaire (JOABPEQ) following decompression surgery for lumbar spinal stenosis. J Clin Neurosci. 2019;69:93-6.

40. Fujimori T, Miwa T, Oda T. Responsiveness of the Japanese Orthopaedic association Back pain evaluation questionnaire in lumbar surgery and its threshold for indicating clinically important differences. Spine J. 2019;19:95-103.

41. Tashjian RZ, Deloach J, Green A, Porucznik CA, Powell AP. Minimal clinically important differences in ASES and simple shoulder test scores after nonoperative treatment of rotator cuff disease. J Bone Joint Surg Am. 2010; 92:296-303.

42. Tashjian RZ, Hung M, Keener JD, Bowen RC, McAllister J, Chen W, Ebersole G, Granger EK, Chamberlain AM. Determining the minimal clinically important difference for the American shoulder and elbow surgeons score, simple shoulder test, and visual analog scale (VAS) measuring pain after shoulder arthroplasty. J Shoulder Elb Surg. 2017;26:144-8.

43. Tashjian RZ, Shin J, Broschinsky K, Yeh CC, Martin B, Chalmers PN, Greis PE, Burks RT, Zhang Y. Minimal clinically important differences in the American shoulder and elbow surgeons, simple shoulder test, and visual analog scale pain scores after arthroscopic rotator cuff repair. J Shoulder Elb Surg. 2020.

44. Norman GR, Stratford P, Regehr G. Methodological problems in the retrospective computation of responsiveness to change: the lesson of Cronbach. J Clin Epidemiol. 1997;50:869-79.

45. Fritz JM, Irrgang JJ. A comparison of a modified Oswestry low Back pain disability questionnaire and the Quebec Back pain disability scale. Phys Ther. 2001;81:776-88.

\section{Publisher's Note}

Springer Nature remains neutral with regard to jurisdictional claims in published maps and institutional affiliations.

Ready to submit your research? Choose BMC and benefit from:

- fast, convenient online submission

- thorough peer review by experienced researchers in your field

- rapid publication on acceptance

- support for research data, including large and complex data types

- gold Open Access which fosters wider collaboration and increased citations

- maximum visibility for your research: over $100 \mathrm{M}$ website views per year

At $\mathrm{BMC}$, research is always in progress.

Learn more biomedcentral.com/submissions 